\title{
A dentist's guide to using the BNF: part II
}

- This paper illustrates how the BNF can facilitate rational prescribing.

- It explains how to select effective drug treatments, minimise harm in patients with co-morbidities and minimise drug interactions and adverse drug reactions.

\author{
D. Wray and S. M. S. Wagle ${ }^{2}$
}

- It explains how to select drug treatment in special patient groups.

- It explains how to choose the right dose.

- It provides advice on when it is appropriate to prescribe an unlicensed medicine.

The BNF can be used as a tool to facilitate rational prescribing. In the second of this two-part series, we describe how dentists can maximise the effectiveness of their prescribing and minimise the risks involved while tailoring medicines to the individual needs of each patient.

\section{USING THE BNF AS A TOOL FOR RATIONAL PRESCRIBING}

The rational use of medicines involves striking the balance between:

- Maximising effectiveness

- Minimising risk

- Respecting patient choice, and

- Minimising cost.

In this second article of a two-part series we describe how the British National Formulary $(B N F)^{2}$ can be used as a tool to achieve rational prescribing.

\section{Identifying effective drug treat- ments}

The prescribing notes in the $B N F$ provide an overview of the drug management of common oral conditions and allow you to appraise treatment options rapidly. For example, Table 1 in chapter 5 provides advice on the initial antibacterial treatment of various oral infections (Fig. 1). However, to select safe and effective drug treatments in individual patients, you need to use this type of information in conjunction with prescribing details

'Chairman of BNF Dental Advisory Group/Professor of Oral Medicine, Glasgow Dental Hospital and School, 378 Sauchiehall Street, Glasgow, G2 3JZ; ${ }^{2 *}$ Assistant Editor, BNF Publications, Royal Pharmaceutical Society of Great Britain, 1 Lambeth High Street, London, SE1 7JN ${ }^{*}$ Correspondence to: Shama Wagle swagle@bnf.org

\section{Refereed Paper}

DOI: 10.1038/sj.bdj.2008.347

${ }^{\circledR}$ British Dental Journal 2008; 204: 487-491 of the drugs and a knowledge of the patient's medical and drug history.

\section{Minimising drug hypersensitivity reactions}

Prescribing a drug for which an allergy already exists is recognised as a cause of fatal prescribing error. ${ }^{3}$ You should ask if the patient has any drug allergies and record them carefully (see Example 1).

\section{Example 1}

You see a woman with a facial swelling due to a periapical abscess. She informs you that she developed acute breathing problems and a low blood pressure when given piperacillin injection.

Which antibiotic should you prescribe?

The prescribing notes in Table 1, section 5.1 of the $B N F($ Fig. 1) show that possible options for treatment include amoxicillin or metronidazole.

The BNFshows that piperacillin is a penicillin. You can use the prescribing notes on penicillins in section 5.1.1 of the $B N F$ to determine whether your patient is allergic to penicillin.

The patient has a history of immediate hypersensitivity reaction to piperacillin. Patients who are allergic to one penicillin will be allergic to all because the hypersensitivity is related to the basic penicillin structure. You should avoid prescribing amoxicillin for this patient. Metronidazole can be used.

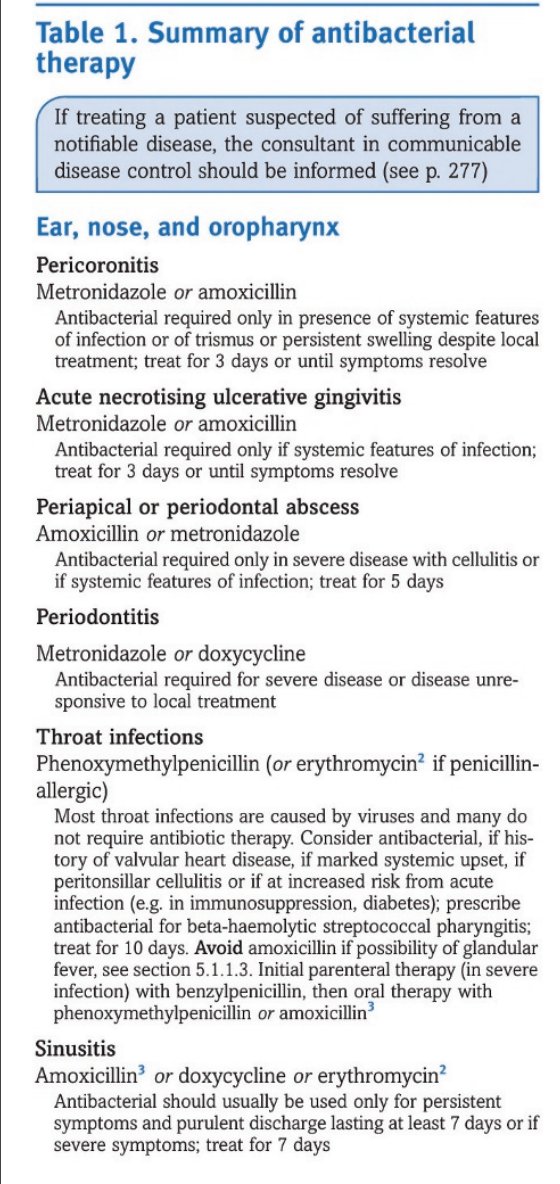

Fig. 1 The prescribing notes in the $B N F$ provide an overview of the drug management of common oral conditions, allowing rapid appraisal of treatment options

\section{Minimising harm in patients with co-morbidities}

The drug you choose to treat an oral condition should have minimal detrimental effects on the patient's other diseases and minimise the patient's susceptibility 
to adverse effects. To achieve this, you need to review the cautions and contra-indications of the drug you intend to prescribe. This information can usually be found in the drug monograph. However, if a class of drugs, such as the tetracyclines, share the same cautions and contra-indications, these are amalgamated in the prescribing notes, while those unique to a particular tetracycline are included in its individual monograph (Fig. 2).

\section{Example 2}

You see a woman with severe periodontitis who requires adjunctive antibiotic therapy. She also has a history of systemic lupus erythematosus.

Which antibiotic should you prescribe?

The prescribing notes in Table 1, section 5.1 of the BNF(Fig. 1) show that possible options for treatment include metronidazole or doxycycline.

The cautions for tetracyclines show that they may exacerbate systemic lupus erythematosus. Metronidazole can be used.

\section{Example 3}

You see a man with severe periodontitis who requires adjunctive antibiotic therapy. He also has problems with alcohol dependence.

\section{Which antibiotic should you prescribe?}

Doxycycline (Fig. 2) should be used with caution in patients with alcohol dependence because the serum-doxycyline concentration may fall below minimum therapeutic concentrations in these patients. This is not a class effect shared by other tetracyclines. Metronidazole may produce a disulfiram-like reaction with alcohol. Either tetracycline or oxytetracycline can be prescribed for this patient.

Examples 2 and 3 illustrate how the drug treatment of severe periodontitis varies according to the patient's co-morbidities. The information under Cautions allows you to assess the risks of using a drug in a patient with co-morbidities that are also included in the Cautions for that drug - if a safer alternative cannot be found, the drug may be prescribed while monitoring the patient for adverse effects or deterioration in the co-morbidity. Contra-indications are far more restrictive than Cautions and mean that the drug should be avoided in a patient with a contra-indicated condition. For example, non-steroidal anti-inflammatory drugs (NSAIDs) should be avoided in patients with severe heart failure.

\section{Minimising harm in patients with hepatic or renal impairment}

Hepatic or renal disease may alter the metabolism or excretion of some drugs. Hepatic reserve and renal clearance reduce with age, and the elderly are particularly susceptible to the side-effects of drugs that are eliminated by these routes. Appendices 2 and 3 in the BNF provide guidance on drugs to be avoided or used with caution in hepatic or renal impairment (see Examples 4-6). You should aim to:

- Select drug treatments that minimise the potential for drug accumulation, adverse drug reactions and exacerbation of pre-existing liver or renal disease

- Make appropriate drug dose adjustments for these patients

- Monitor patients appropriately if it is necessary to prescribe drugs whose response is altered by liver or renal disease.

In the Appendices of the print version of the $B N F$, drugs are included in alphabetical order. In the digital versions, this information can be accessed directly from hyperlinks located in the drug monographs.

\section{Example 4}

What dose of aciclovir should be prescribed for a man with oral lesions associated with herpes zoster? He has a history of chronic renal impairment and his records show that his eGFR two weeks ago was $22 \mathrm{ml} /$ minute $/ 1.73 \mathrm{~m}^{2}$.

According to the record for aciclovir in Appendix 3 of the $B N F$, the normal dose for herpes zoster should be given every eight hours if the creatinine clearance

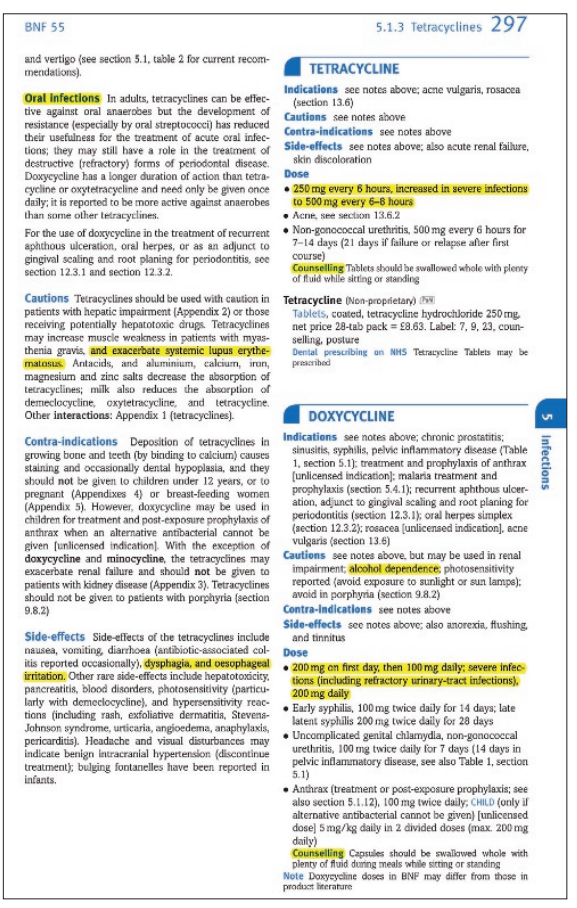

Fig. 2 The cautions, contra-indications and side-effects shared by all the tetracyclines are amalgamated in the prescribing notes while those unique to a particular tetracycline are included in its individual monograph. The monographs for tetracycline and doxycycline include counselling advice on administration. As no specific dose of tetracycline or doxycycline is given for the dental indication, the general dose can be used

is $10-25 \mathrm{ml} /$ minute. For this patient of average build and height, the eGFR can be used in place of creatinine clearance to make drug dose adjustments. This patient should receive aciclovir $800 \mathrm{mg}$ every eight hours for seven days.

\section{Example 5}

You are thinking of prescribing an NSAID for a woman with postoperative dental pain. She has a history of chronic renal impairment and her most recent eGFR was $55 \mathrm{ml} /$ minute $/ 1.73 \mathrm{~m}^{2}$. Why might an NSAID not be the most appropriate analgesic for this patient?

According to the record for NSAIDs in Appendix 3 of the BNF, an NSAID may cause sodium and water retention and further deterioration in renal function in this patient. It is more appropriate to try paracetamol first. An NSAID should only be used, if essential, at the lowest effective dose and renal function should be monitored. 


Example 6
What dose of metronidazole should
be prescribed for a woman with acute
necrotising ulcerative gingivitis? She
also has severe primary biliary cirrhosis.
According to the record for metro-
nidazole in Appendix 2 of the $B N F$, in
severe liver disease the total daily dose
of metronidazole should be reduced to
one-third, and given once daily. This
patient should receive 200 mg daily for
three days.

\section{Minimising harm during pregnancy and breast-feeding}

Appendices 4 and 5 in the BNF provide guidance on drugs to be avoided or used with caution during pregnancy or breast-feeding (see Examples 7 and 8). You should aim to:

- Determine whether a mother's condition requires drug therapy

- Select drug treatments that minimise harm to the foetus, nursing infant and mother

- Monitor the infant for potential sideeffects of drugs used by the mother during pregnancy or breast-feeding.

\section{Example 7}

A woman who is 32 weeks pregnant presents with acute dental pain. How should you treat this patient's pain?

According to the record for NSAIDs in Appendix 4 of the BNF, if an NSAID is used regularly during the third trimester of pregnancy, it may cause closure of the foetal ductus arteriosus in utero and possibly pulmonary hypertension of the newborn. The onset of labour may be delayed and the duration of labour may be increased. Paracetamol is not known to be harmful and can be used.

\section{Example 8}

A woman presents with oropharyngeal candidiasis that has not responded to a seven day course of nystatin suspension. She is breast-feeding a sevenmonth-old healthy baby girl. How should you treat this patient's oral thrush?
According to the prescribing notes in section 5.2 and section 12.3 .2 of the BNF, fluconazole is effective for infection that has not responded to topical therapy. The record for fluconazole in Appendix 5 shows that although it is present in milk, the amount is probably too small to be harmful. Before prescribing the fluconazole, you should also consider the health status of the nursing infant, in this case a healthy baby.

\section{Minimising drug interactions}

Details of drug interactions can be found in Appendix 1 of the print version of the BNF. Drugs are listed in alphabetical order. The symbol • is placed against interactions that are potentially hazardous and where combined administration of the drugs should be avoided (or only undertaken with caution and appropriate monitoring) (Fig. 3). Interactions that have no symbol do not usually have serious consequences.

In the digital versions, you can search for interactions between a combination of drugs using the Interactions search facility (Fig. 4). Alternatively, interactions for a specific drug can be accessed from a hyperlink in the drug monograph. Potentially hazardous drug interactions are shown in bold on a pink background while those without serious consequences are shown on a beige background.

Examples 9 and 10 show how use of the BNF can help prescribing in cases with potential for drug interaction.
Example 9

A 23-year-old man presents with severe symptoms of sinusitis and purulent discharge that started nine days ago. He is being treated with isotretinoin capsules for acne. Which antibiotic should you prescribe for his sinusitis?

The prescribing notes in Table 1, section 5.1 of the $B N F($ Fig. 1) show that possible options for treatment include amoxicillin doxycycline, or erythromycin.

According to Appendix 1, isotretinoin is a retinoid. There is a possible increased risk of benign intracranial hypertension when retinoids are given with tetracyclines. It is classified as a potentially hazardous interaction and concomitant use should be avoided.

Amoxicillin or erythromycin can be prescribed because the BNF does not include an interaction between these antibiotics and retinoids.

\section{Remifentanil see Opioid Analgesics Repaglinide see Antidiabetics Retinoids \\ Alcohol: etretinate formed from acitretin in presence of alcohol \\ - Antibacterials: possible increased risk of benign intra- cranial hypertension when retinoids given with -tetracyclines (avoid concomitant use) \\ - Anticoagulants: acitretin possibly reduces anticoagulant effect of -coumarins \\ Antiepileptics: isotretinoin possibly reduces plasma concentration of carbamazepine \\ - Cytotoxics: acitretin increases plasma concentration of -methotrexate (also increased risk of hepatotoxi- city)—avoid concomitant use}

Fig. 3 The symbol $\bullet$ is placed against interactions that are potentially hazardous and where combined administration of the drugs should be avoided (or only undertaken with caution and appropriate monitoring)

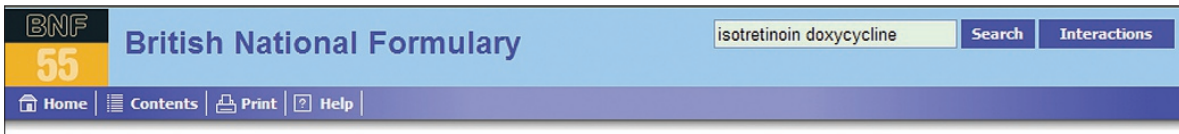

Home $>$ British National Formulary

\section{Interactions Search Results}

Find interactions between isotretinoin and doxycycline.

Eisotretinoin

tidoxycycline

Interaction search: there is 1 document listing interactions between pairs of interactants searched.

\section{tetracyclines + retinoids}

potentially hazardous

Accessibility I Terms and Conditions

Fig. 4 In the digital versions of the $B N F$, you can search for interactions between a combination of drugs using the 'Interactions' search facility 


Example 10
You see a man with pericoronitis
that persists despite local treatment.
The patient has a mitral valve prosthe-
sis and takes warfarin. His anticoagu-
lant record shows that his INR is
stable at 3.5. His next appointment
at the anticoagulant clinic is in four
weeks time.
Which antibiotic should you prescribe?
The prescribing notes in Table 1, section
5.1 of the BNF(Fig. 1) show that possible
options for treatment include metronida-
zole or amoxicillin.
According to Appendix 1, metroni-
dazole may enhance the anticoagulant
effect of warfarin and this is classified
as a potentially hazardous interaction.
Although studies have failed to demon-
strate an interaction between warfarin
and broad-spectrum penicillins such
as amoxicillin, common experience in
anticoagulant clinics is that the INR can
be altered by these antibiotics.
Amoxicillin can be prescribed but you
should arrange for the patient to be seen
by the anticoagulant clinic earlier than
scheduled. The patient should be advised
to seek medical attention if persistent
bleeding occurs.

\section{Side-effects}

The BNF includes clinically relevant side-effects for most drugs. Recognising that hypersensitivity reactions can occur with virtually all medicines, this effect is not generally listed, unless the drug carries an increased risk of such reactions. The $B N F$ also omits effects that are likely to have little clinical consequence (eg transient increases in liver enzymes).

Side-effects are generally listed in order of frequency and arranged broadly by body systems. Occasionally rare sideeffects might be listed first if it is considered to be important because of its seriousness.

When choosing a drug you should review the impact that any potential side-effect may have on a patient's quality of life. In other words, you need to assess the risks and benefits of prescribing a particular drug (see Example 11).

\section{Example 11}

A man is referred to you because he has difficulty swallowing and severe periodontitis which requires adjunctive antibiotic therapy. Which antibiotic should you prescribe for his periodontitis?

The side-effects for tetracyclines (Fig. 2) show that they can cause dysphagia and oesophageal irritation. Furthermore, the monographs for doxycycline, tetracycline, and oxytetracycline include counselling advice that recommends that they should be swallowed whole with plenty of fluid while sitting or standing. Metronidazole suspension is more appropriate for this patient.

\section{Selecting the right dose and route}

The dose is located in the Dose section of the monograph or preparation entry in the BNF. The doses of some drugs vary according to indications - if no specific dose is given for a dental indication then the general dose can be used. The dose for children will vary according to their age or body-weight. You may need to adjust the dose of some drugs if their effects are altered by concomitant use with other drugs, or in patients with hepatic or renal impairment. Where a drug can be administered by more than one route you should ensure that you choose the right dose for the right route (see Example 12).

\section{Example 12}

What dose of doxycycline should you prescribe for a 37-year-old man with severe symptoms of sinusitis that is not responding to local treatment?

The general dose for doxycycline in section 5.1.3 of the BNF, $200 \mathrm{mg}$ on the first day then $100 \mathrm{mg}$ daily, can be used. The lower dose of doxcycline given in section 12.3.1 does not have an antibacterial effect.

\section{Minimising antibiotic resistance}

To minimise the development of resistant organisms you should:

- Select an antibiotic with a spectrum of activity that is appropriate for the infection. Regional guidelines and local patterns of resistance will influence your treatment

- Use adequate doses of an antibiotic

- Avoid unduly prolonged courses of antibiotics.

\section{Finding a suitable formulation}

You should prescribe a formulation that is suitable for your patient and that fits in with their daily routine. Use sugarfree preparations where possible.

\section{Involving the patient}

The whole prescribing process will be a waste of time if your patient does not take the medicine you have prescribed. You should agree the health outcomes and the strategy for achieving them with your patient. You should also take the time to explain the rationale and the potential adverse effects of treatment.

\section{Writing legible prescriptions}

The section on 'Prescription Writing' is located near the front of the BNF under 'General Guidance on Prescribing'. It provides useful tips on writing clear prescriptions that will minimise dispensing and administration errors.

\section{Monitoring drug treatment}

You need to ensure that the treatment you prescribe is achieving the expected benefits, while monitoring the patient for unwanted side-effects. Patients are often the first to notice some of these changes and, if possible, you should involve them in the monitoring process.

\section{Reporting adverse drug reactions}

All adverse drug reactions (ADRs) to newly licensed medicines and serious adverse drug reactions to established medicines should be reported to the MHRA on the yellow cards supplied with the print version of the BNF, or online at www.yellowcard.gov.uk. (see Example 13).

\section{Example 13}

You see a patient who is complaining of dry mouth that started one week ago and is not relieved by simple measures. He is trying to stop smoking and was started on varenicline two weeks ago. 
Reviewing the side-effect profile in the $B N F$, you find that dry mouth is common with varenicline. You suspect that varenicline is causing this patient's dry mouth. Should you report this to the regulatory authorities?

According to the preparation entry for this drug in the $B N F$, varenicline is assigned a black triangle $(\boldsymbol{\Delta})$ symbol. According to the section on 'Adverse Reactions to Drugs' (located near the front of the BNFunder 'General Guidance on Prescribing'), this means that it is a newly licensed medicine that is being monitored intensively by the MHRA. For medicines showing the black triangle symbol, the MHRA asks that all suspected reactions (including those considered not to be serious) should be reported, even if it is not certain that the drug has caused it, or if the reaction is well recognised, or if other drugs have been given at the same time.

You should report this suspected adverse drug reaction on the yellow card and you should inform the patient's general practitioner of the action you have taken.

\section{Unlicensed medicines}

The BNF includes unlicensed use of medicines when the clinical need cannot be met by licensed medicines; such use should be supported by appropriate evidence and experience. For example, the BNF recommends the unlicensed use of buccal midazolam for the emergency treatment of status epilepticus in dental practice. The alternative licensed drugs are not practical for dentists to administer by intravenous injection or by the rectal route.

\section{FINAL REMARKS}

We encourage you to become familiar with the information on drugs that you prescribe and encounter frequently, and to apply the principles of rational prescribing when choosing drug treatment. In this way the complexity of the prescribing process can be eased.

We thank L. Oliver, P. Wilder and P. Lee for preparing images of the $B N F$.

1. Barber N. What constitutes good prescribing? BMJ 1995; 310: 923-925

2. Joint Formulary Committee. British National Formulary. 55th ed. London: British Medical Association and Royal Pharmaceutical Society of Great Britain, 2008.

3. Patient Safety Observatory. Safety in doses: medication safety incidents in the NHS. London: National Patient Safety Agency, 2007. 